\title{
Escoamento na esteira de turbinas eólicas: Análise espectral da turbulência mediante testes em túnel de vento
}

Flow in the wake of wind turbines: Turbulence spectral analysis by wind tunnel tests

\author{
Adrián Roberto Wittwer ${ }^{1}$, Rodrigo Dorado ${ }^{2}$, Gervásio Annes Degrazia ${ }^{3}$, Acir Mércio \\ Loredo-Souza ${ }^{4}$, Bardo Ernst Josef Bodmann ${ }^{5}$
}

\author{
${ }^{1}$ Facultad de Ingeniería, Universidad Nacional del Nordeste, Resistencia, Argentina \\ a_wittwer@yahoo.es; acir@ufrgs.br; bejbodmann@gmail.com \\ 2,45 Universidade Federal de Rio Grande do Sul, Porto Alegre, RS, Brasil \\ dorado@ste-pdi.com.br \\ ${ }^{3}$ Universidade Federal de Santa Maria RS, Brasil \\ gervasiodegrazia@gmail.com
}

\begin{abstract}
Resumo
A interação entre o vento incidente e os aerogeradores em um parque eólico provoca déficit de velocidade, no caso de geradores sucessivos, e aumento nos níveis de turbulência na esteira das turbinas eólicas. O escoamento turbulento é caracterizado pela superposição de esteiras. Neste trabalho, a técnica de avaliação espectral da turbulência em modelos a escala de aerogeradores em um túnel de vento de camada limite é apresentada, e varias medições das flutuações de velocidade são analisadas. Os resultados permitem avaliar a configuração dos espectros nos diferentes rangos de freqüência e as diferenças no comportamento espectral do vento incidente e do escoamento na esteira da turbina.
\end{abstract}

Palavras-chave: Aerogerador. Esteira turbulenta. Modelos em escala reduzida.

\begin{abstract}
The interaction between the incident wind and wind turbines in a wind farm causes mean velocity deficit and increased levels of turbulence in the wake. The turbulent flow is characterized by the superposition of the wind turbine wakes. In this work, the technique of turbulence spectral evaluation to reduce scale models in a boundary layer wind tunnel is presented, and different measurements of velocity fluctuations are analyzed. The results allow evaluating the spectrum configuration at different frequency ranges and the differences of the spectral behavior between the incident wind and the turbine wake flow.
\end{abstract}

Keywords: Wind turbine. Turbulent wake. Reduced scale models. 


\section{Introdução}

Existem diferentes aspectos a serem considerados na análise da interação fluidoestrutura entre o vento incidente $\mathrm{e}$ os aerogeradores em um parque eólico. Dentro deles temos o déficit de velocidade no caso de geradores sucessivos e os níveis de turbulência na esteira das turbinas eólicas. Em um parque de aerogeradores, o escoamento turbulento é caracterizado pela coexistência e superposição de múltiplas esteiras e as perdas do potencial eólico, produto dos efeitos de esteira, podem alcançar até o $20 \%$ do total da energia. Alguns trabalhos mostram que aerogeradores operando dentro de um parque podem ter uma queda na potência gerada de até $40 \%$, quando comparada com o caso da turbina independente (Crespo et al., 1999, Frandsen et al., 2004). Outras investigações analisam a degradação da potência que se produze a partir da segunda fila dos aerogeradores e qual é sua evolução com relação a primeira fila (Barthelmie et al., 2007).

Os problemas de fatiga nas turbinas estão associados à intensidade de turbulência que também tem incidência direta nas forças e momentos atuantes nas palas do dispositivo de geração. Ainda que existam trabalhos de pesquisa, de tipo experimental e computacionais, destinados a melhorar a compreensão destes problemas, não existem modelos suficientemente confiáveis para predizer a distribuição espacial da turbulência nos parques eólicos.

As dificuldades que implicam a coexistência de esteiras múltiplas e superpostas, os efeitos camada limite, a topografia local, os níveis de turbulência e a estratificação térmica sugerem a utilização complementária de distintos tipos de abordagem para a caracterização do escoamento turbulento nos parques eólicos. A experimentação em túnel de vento utilizando modelos a escala reduzida permite reproduzir os fenômenos característicos da interação sob condições controladas. Existem trabalhos centrados na simulação física de escoamentos na camada limite (Liu et al., 2003), na estrutura da esteira dos aerogeradores (Bartl, 2011), e outros que avaliam a turbulência efetiva de um parque eólico (Cal et al., 2010, Chamorro e Porté-Agel, 2011, Henriksen et al., 2012). Em geral, este tipo de estudo permite determinar que os modelos básicos de análise superestimam a eficiência dos parques, sendo que calculam esteiras com extensões menores às reais.

Este trabalho é um estudo experimental preliminar das características espectrais da turbulência na esteira de um aerogerador. Medições da componente longitudinal da velocidade flutuante foram realizadas no escoamento incidente e na esteira do modelo reduzido de uma turbina eólica na seção de testes de um túnel de vento. A adequação da técnica espectral e as mudanças na configuração dos espectros da turbulência no vento incidente e na esteira são analisadas a partir de estes experimentos preliminares.

\section{Parâmetros característicos do escoamento em um aero-gerador}

Quando o vento aproxima-se ao rotor do aero-gerador ocorre uma diminuição da componente axial da velocidade antes do que o ar passe a través do plano do rotor. Esta redução constitui o déficit de velocidade axial e define o fator de indução axial:

$$
a=\frac{U_{\infty}-U_{1}}{U_{\infty}}
$$

onde $U_{\infty}$ é a velocidade da corrente livre e $U_{1}$ a velocidade no plano do rotor. Depois de atravessar o plano do rotor, a componente axial da velocidade diminui mais ainda e, considerando uma análise unidimensional simplificado, torna-se $U_{\infty(1-2 a)}$ na posição longe a sotavento. Quando o escoamento atravessa o rotor, ocorre uma queda de pressão. A diminuição da velocidade do escoamento a sotavento do rotor se corresponde com a recuperação da pressão até atingir novamente o valor da pressão atmosférica. Nesta idealização, se considera que a metade da indução ocorre antes do rotor e a segunda metade da indução ocorre depois do rotor.

Outro fator usado para descrever o campo de velocidades em torno a um gerador é o fator de indução tangencial $a^{\prime}$, produto da rotação do escoamento na esteira:

$$
a^{\prime}=\frac{\omega}{2 \Omega}
$$


onde $\Omega$ é a velocidade angular do rotor e $\omega$ a velocidade de rotação da esteira. Valores elevados de $a^{\prime}$ indicam que a potência produzida será baixa e uma importante quantidade de energia cinética associada à rotação da esteira.

O fator de indução axial $a$ está diretamente relacionado à potência extraída pela turbina. Se o vento incidente na turbina para completamente pela ação das palas da turbina $(a=1)$, não existiria extração de potência sendo que não haveria escoamento a través do plano do rotor. Por outro lado, se não existe um cambio na velocidade do vento ultrapassando as palas $(a=0)$ não haveria extração de potência, sendo que a energia cinética mantém-se constante antes e depois do rotor. A potência extraída pela turbina é definida por:

$$
W=\frac{1}{2} \rho A U_{\infty}^{3} 4 a(1-a)
$$

onde $\rho$ é a densidade do ar e A área do rotor. A potência do vento incidente é:

$$
W_{v i}=\frac{1}{2} \rho A U_{\infty}^{3}
$$

O coeficiente de potência $C p$ é a fração da potência do vento incidente extraída pelo rotor $\left(W / W_{v i}\right)$.

Finalmente, existe outro parâmetro importante na análise das características de operação de um aero-gerador denominado relação de velocidade no extremo da pala (tipspeed ratio $\lambda$ ). É definido como a relação entre a velocidade tangencial na borda da pala e a velocidade de corrente livre:

$$
\lambda=\frac{\Omega r}{U_{\infty}}
$$

onde $r$ é o radio do rotor. A gráfica do coeficiente de potência $C p$ em função de $\lambda$ é a curva característica de uma turbina deste tipo.

\section{Dispositivo experimental e instrumentação}

Os experimentos foram desenvolvidos no túnel de vento "Jacek P. Gorecki" da UNNE,
Argentina (Wittwer e Möller, 2000). A Figura 1 mostra os dispositivos de simulação da camada limite atmosférica (CLA) e do modelo da turbina na seção de testes do túnel de vento. Todas as medições de componente longitudinal de velocidade flutuante $u$ foram realizadas com a simulação da CLA neutra obtida pelo método de Counihan utilizando rugosidade superficial e dispositivos de mistura (Figura 1). As características do vento incidente simulado no túnel correspondem a um perfil da lei de potência com expoente $\alpha=0.27$ e altura gradiente $z_{g}=1.20 \mathrm{~m}$. Para a medição da velocidade de vento nos túneis foi utilizado um anemômetro de fio quente (Figura 2). O modelo da turbina eólica corresponde a um aerogerador UNIPOWER de 3 palas, de $100 \mathrm{~m}$ de altura da torre e $100 \mathrm{~m}$ de diâmetro de rotor (Figura 3). A escala do modelo é aproximadamente $1 / 450$, assim a altura máxima do modelo é $0.33 \mathrm{~m}$. A Figura 4 simplesmente mostra o modelo da turbina girando pela ação do vento incidente.

As medições da velocidade $u$ foram realizadas a três alturas distintas $z=0.17,0.225 \mathrm{e}$ $0.28 \mathrm{~m}$, primeiro com vento incidente, e logo depois, na esteira do modelo do aerogerador. À distância a sotavento do modelo é $x=0.225 \mathrm{~m}$. As series numéricas representativas da velocidade $\mathrm{u}$ foram obtidas com uma freqüência de amostragem de $1024 \mathrm{~Hz}$ durante 128 segundos. Antes da aquisição das amostras, um filtro eletrônico passa-baixos foi ajustado a 0.3 $\mathrm{kHz}$.

O valor do número de Reynolds

$$
\operatorname{Re}=\frac{\rho \bar{U} \phi_{g}}{\mu}
$$

definido com relação à velocidade média dos testes $\bar{U}$ e o diâmetro do gerador $\emptyset_{g}$ é $1.63 \times 10^{5}$, sendo $\mu$ a viscosidade absoluta do ar. A velocidade de rotação da turbina foi estimada pela análise de imagens e se manteve aproximadamente constante durante as medições, mas resulta importar salientar que não foi conseguido atingir os valores da relação adimensional de velocidades $\lambda$ para garantir a semelhança do fenômeno no modelo reduzido. 


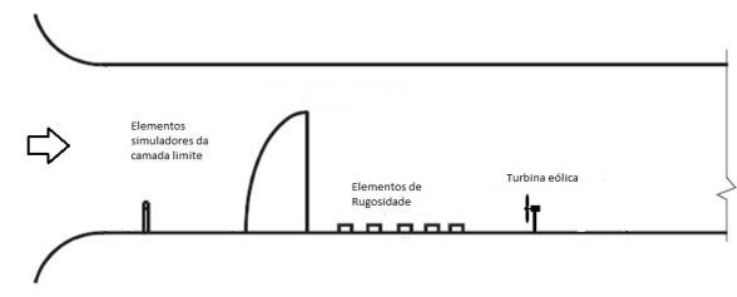

Figura 1 - Esquema dos dispositivos de simulação da CLA e do modelo da turbina no túnel de vento

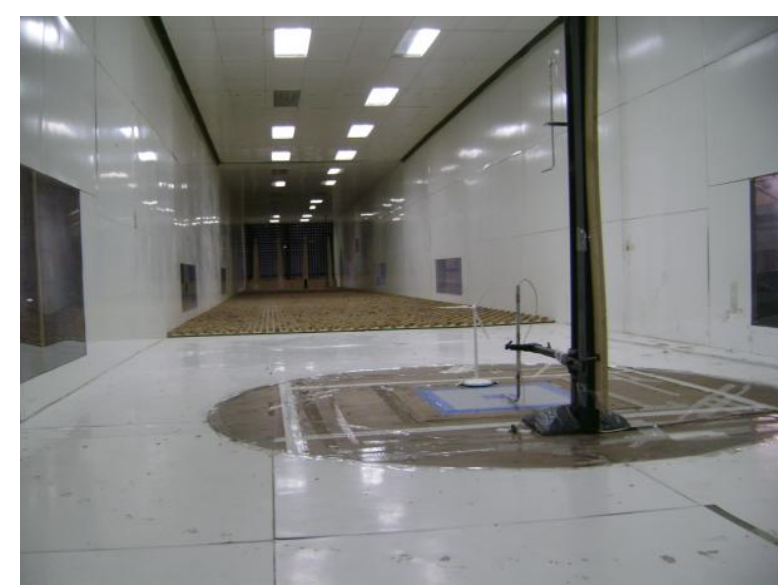

Figura 2 - Vista da seção de testes, do modelo da turbina eólica e do aparelho de medição

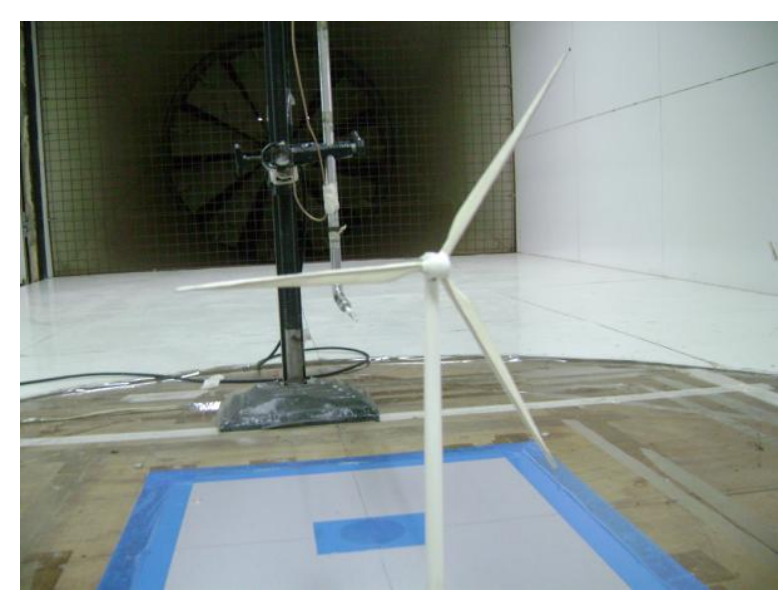

Figura 3 - Detalhe do modelo da turbina eólica

\section{Resultados}

Nesta seção são apresentados os resultados obtidos nos testes realizados. As principais características das medições (altura $z$, velocidade média $\bar{U}$ e variância da flutuação de velocidade $\left.\sigma u^{2}\right)$ são indicadas na Tabela 1. A seguir, os espectros $\mathrm{Su}$ correspondentes à componente longitudinal das flutuações de velocidade são apresentados. A função de densidade autoespectral ou espectro de potência representa a variação em função da freqüência, do valor quadrático médio da flutuação de velocidade em função do tempo $u(t)$ fornecida por uma serie numérica contínua adquirida com um intervalo de tempo $t$, e fica expressa por:

$$
S_{u}(f)=\frac{1}{B_{e} T} \int_{0}^{T} u^{2}\left(f, B_{e}, t\right) d t
$$

onde $B_{e}$ é a largura de banda (resolução do espectro), e $\mathrm{T}$ o tempo de integração adequado. A integral desta função permite obter a variância da flutuação de velocidade $\sigma u^{2}$.

Primeiro são avaliados os espectros com relação ao numero de blocos, promédio e resolução apenas em uma posição $(z=0.17 \mathrm{~m})$. Então, uma comparação entre os espectros obtidos para o vento incidente e na esteira da turbina.

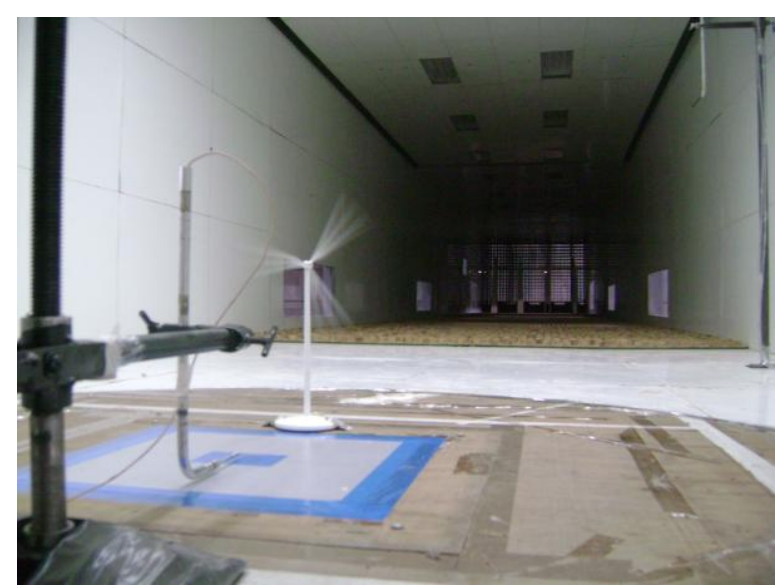

Figura 4 - Vista do modelo da turbina em funcionamento pela ação do vento

\subsection{Resolução dos espectros}

Nas Figuras 5, 6 e 7, com base em uma mesma medição foram obtidos três espectros na posição $z=0.17 \mathrm{~m}$ para o vento incidente. O primeiro é orientado a descrever as baixas freqüências e foi obtido utilizando 8 blocos de 16384 valores cada (Figura 5). O segundo, obtido a partir de 32 blocos de 4096 valores, descreve as freqüências intermédias e altas (Figura 6). Finalmente, o espectro descritivo das altas freqüências foi obtido utilizando 256 blocos de 512 valores (Figura 7).

De forma semelhante foram obtidos os espectros correspondentes à posição $z=0.17 \mathrm{~m}$ para o escoamento na esteira apresentados nas Figuras 8, 9 e 10 e que são descritivos das 
freqüências baixas, intermédias e altas, respectivamente.

Esta avaliação foi realizada para ter uma impressão geral do comportamento espectral em tudo o rango de freqüências. Aumentando o número de blocos, diminui a resolução nas baixas freqüências, mas a média dos blocos permite visualizar melhor o que acontece nas freqüências intermédias e altas. Em particular, para as medições realizadas, permite determinar que a configuração dos espectros nas baixas freqüências para o vento na esteira não apresenta alterações significativas e, por tanto, a análise comparativa será realizada apenas pelos espectros descritivos das altas freqüências.

Tabela 1 - Parâmetros característicos das medições

\begin{tabular}{lcrc}
\hline Medição & $z[\mathrm{~m}]$ & $U[\mathrm{~m} / \mathrm{s}]$ & $\sigma u^{2}\left[(\mathrm{~m} / \mathrm{s})^{2}\right]$ \\
\hline Incidente 170 & 0.170 & 10.49 & 2.32 \\
Incidente 225 & 0.225 & 11.03 & 2.47 \\
Incidente 280 & 0.280 & 11.59 & 2.63 \\
Esteira 170 & 0.170 & 9.29 & 2.36 \\
Esteira 225 & 0.225 & 9.97 & 2.52 \\
Esteira 280 & 0.280 & 11.03 & 2.79 \\
\hline
\end{tabular}

\subsection{Comparação espectral do vento incidente e na estira da turbina}

Finalmente, uma analise comparativa dos espectros obtidos para o vento incidente e na esteira da turbina foi realizada. Na Figura 11, os espectros correspondentes à posição $\mathrm{z}=0.17 \mathrm{~m}$ mostram já algumas diferenças nas freqüências mais baixas e, a partir dos $15 \mathrm{~Hz}$, a separação do espectro na esteira com relação à clara definição da região inercial (pendente -5/3) no espectro do vento incidente. O efeito é previsível a partir da turbulência adicional produzida pela turbina. Entre 200 e $300 \mathrm{~Hz}$, é nitidamente percebido o efeito de filtro passa-baixos.

Na Figura 12, a comparação espectral na posição $\mathrm{z}=0.225 \mathrm{~m}$ (altura do eixo da turbina) indica um comportamento muito semelhante ao anterior, só que nas freqüências mais baixas não se verificam diferenças.

Finalmente, na posição $\mathrm{z}=0.28 \mathrm{~m}, \quad \mathrm{a}$ separação dos espectros é menor que nas duas posições anteriores (Figura 13).

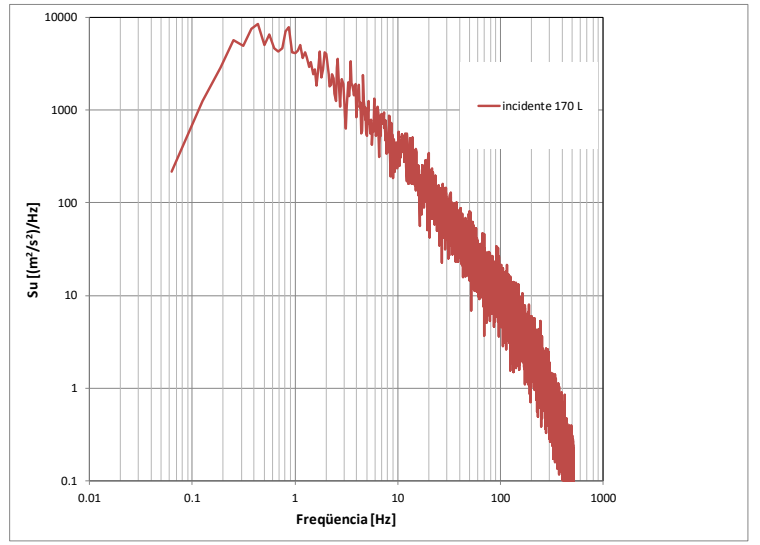

Figura 5: Espectro do vento incidente para $\mathrm{z}=0.17 \mathrm{~m}$ (descrição das baixas freqüências)

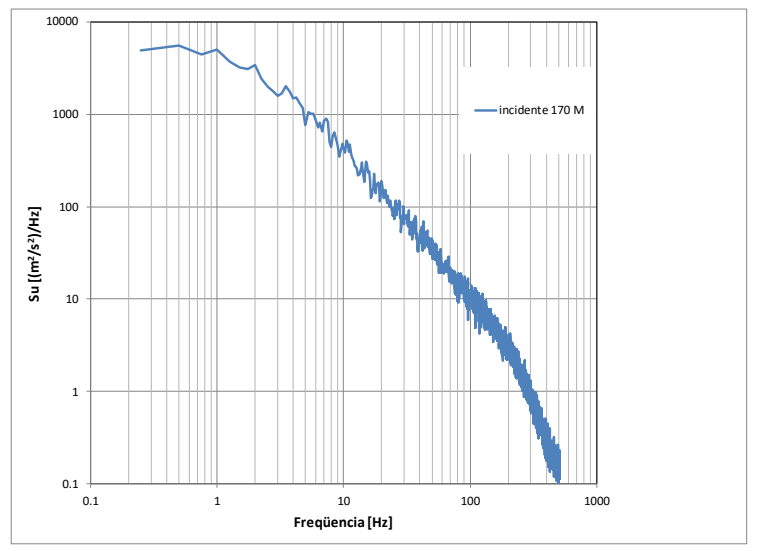

Figura 6 - Espectro do vento incidente para $\mathrm{z}=0.17 \mathrm{~m}$ (descrição das freqüências intermédias e altas)

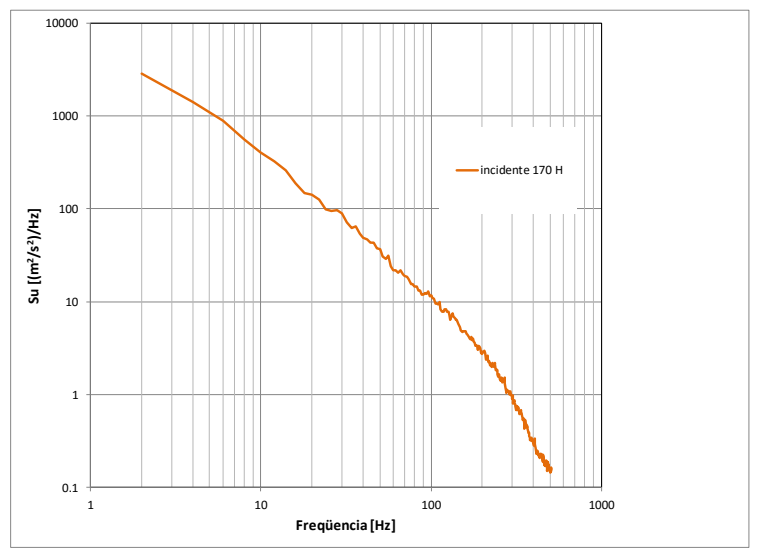

Figura 7 - Espectro do vento incidente para $\mathrm{z}=0.17 \mathrm{~m}$ (descrição das altas freqüências)

Em todas as posições observou-se um aumento da intensidade das flutuações na esteira com relação ao escoamento incidente, não obstante na posição $\mathrm{z}=0.28 \mathrm{o}$ incremento é maior (da ordem de $6 \%$ ). 


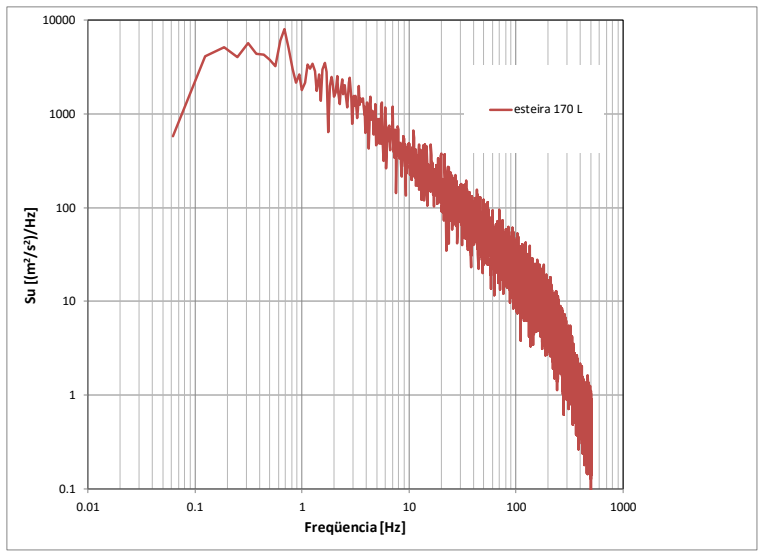

Figura 8 - Espectro do vento na esteira para $\mathrm{z}=0.17 \mathrm{~m}$ (descrição das baixas freqüências)

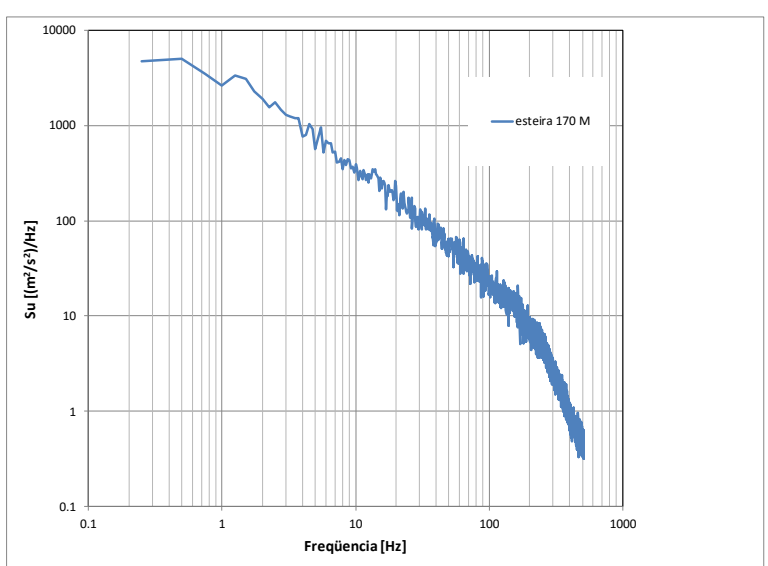

Figura 9 - Espectro do vento na esteira para $\mathrm{z}=0.17 \mathrm{~m}$ (freqüências intermédias e altas)

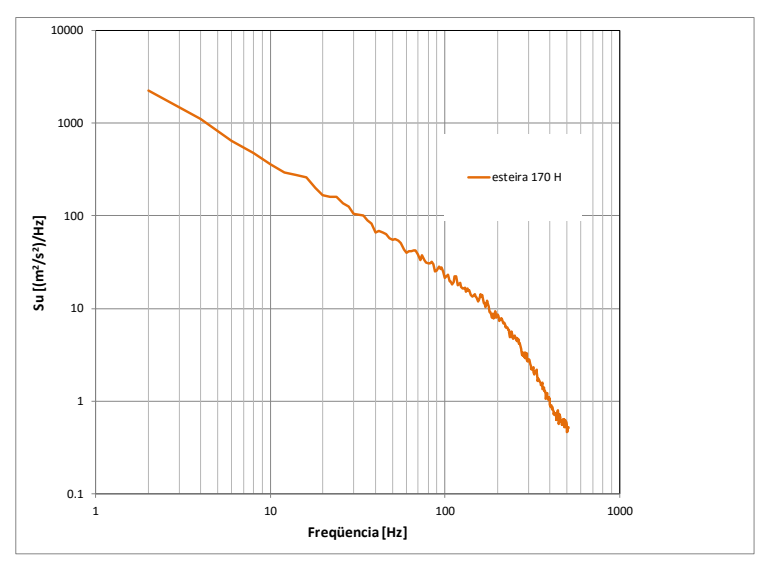

Figura 10 - Espectro do vento na esteira para $\mathrm{z}=0.17 \mathrm{~m}$ (descrição das altas freqüências)

A representação bi logarítmica dos espectros não permite visualizar este comportamento, mas é possível que esse incremento de energia das flutuações fique concentrado nas freqüências menores.

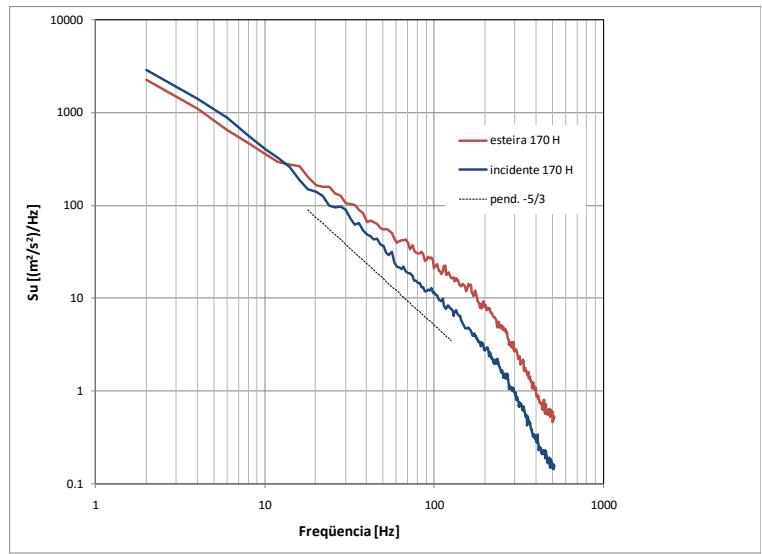

Figura 11 - Comparação espectral do vento incidente e na esteira para $\mathrm{z}=0.17 \mathrm{~m}$

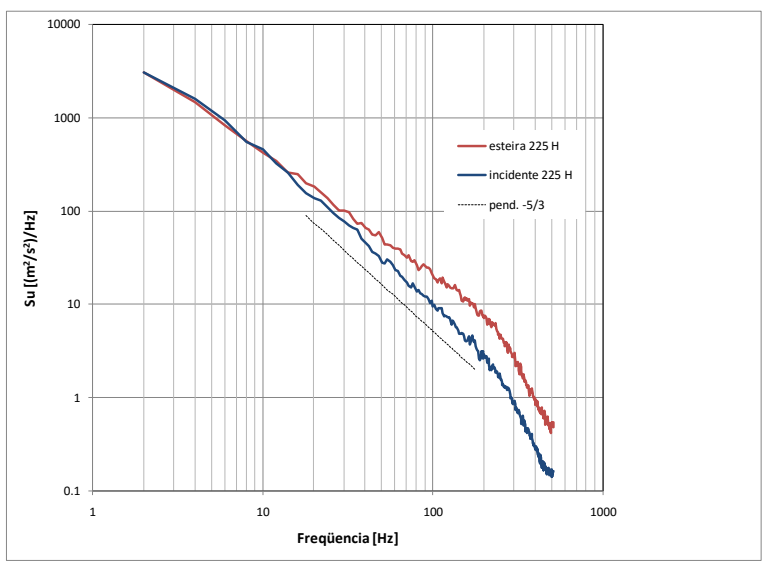

Figura 12 - Comparação espectral do vento incidente e na esteira para $\mathrm{z}=0.225 \mathrm{~m}$

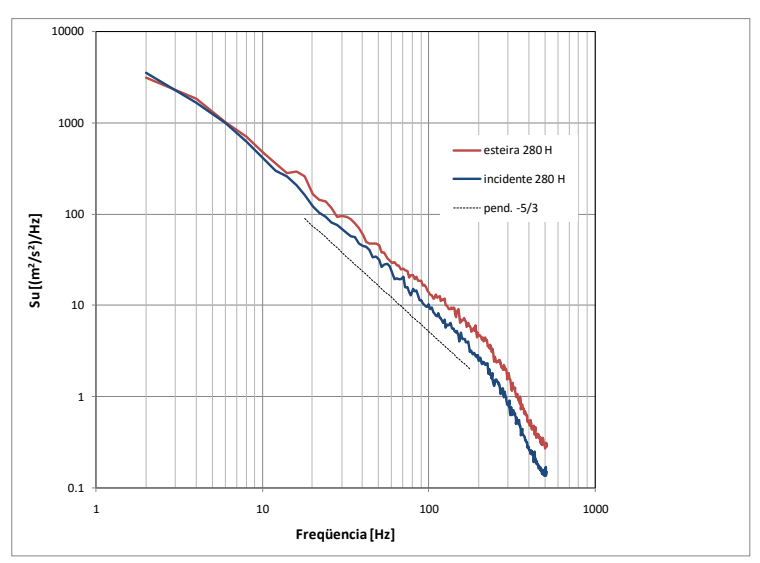

Figura 13 - Comparação espectral do vento incidente e na esteira para $z=0.28 \mathrm{~m}$

\section{Considerações finais}

Neste trabalho é descrita a técnica de avalição espectral da turbulência em modelos reduzidos de aerogeradores em túnel de vento. Uma serie de medições preliminares é analisada visando na configuração dos espectros nos diferentes rangos de freqüência e nas diferenças no 
comportamento espectral do vento incidente e do escoamento na esteira da turbina. A seguir, serão avaliadas as funções de autocorrelação das flutuações de velocidade e serão desenvolvidos novos testes visando atingir os valores da relação adimensional de velocidades que permitam garantir a semelhança do fenômeno, a partir do controle da velocidade do gerador eólico.

\section{Agradecimentos}

Os autores agradecem ao CNPq pelo suporte financeiro para o desenvolvimento deste trabalho.

\section{Referências}

BARTHELMIE, R., RATHMANN, O., FRANDSEN, S., HANSEN, K., POLITIS, PROSPATHOPOULOS, J., RADOS, K., CABEZÓN, D., SCHLEZ, W., PHILLIPS, J., NEUBERT, A., SCHEPERS, J., VAN DER PIJL, S. (2007), Modelling and measurements of wakes in large wind farms, The Science of Making Torque from Wind, Journal of Physics: Conference Series, 75.

BARTL, J. (2011), WAKE MEASUREMENTS BEHIND AN ARRAY OF TWO MODEL WIND TURBINES, Master of Science Thesis, KTH School of Industrial Engineering and Management Energy Technology, EGI-2011-127 MSC EKV 866, Division of Heat and Power Technology, SE-100 44 STOCKHOLM.

CAL, R. B., LEBRÓN, J., CASTILLO, L., KANG, H., MENEVEAU, C. (2010), Experimental study of the horizontally averaged flow structure in a model wind-turbine array boundary layer, JOURNAL OF RENEWABLE AND SUSTAINABLE ENERGY, 2, 013106.

CHAMORRO, L., PORTÉ-AGEL, F. (2011), Turbulent Flow Inside and Above a Wind Farm: A Wind-Tunnel Study, Energies 2011, 4, 19161936.

CRESPO, A., HERNANDEZ, J., FRANDSEN, S. (1999), Survey of Modelling Methods for Wind Turbine Wakes and Wind Farms, Wind Energy, $2,1-24$.
FRANDSEN, S., BARTHELMIE, R., PRYOR, S., RATHMANN, O.,LARSEN, S., HØJSTRUP, J., NIELSEN, P., THØGERSEN, M. (2004), Analytical modeling of wind speed deficit in large offshore wind farms, EWEC 2004, November 22-25, London, UK.

HENRIKSEN, S., MALCOLM, D., THOMSON, J. (2012), Effective Turbulence in Wind Turbine Site Suitability Assessment, EWEA 2012.

LIU, G., XUAN, J., PARK, S. (2003), A new method to calculate wind profile parameters of the wind tunnel boundary layer, Journal of Wind Engineering and Industrial Aerodynamics, 91, 1155-1162.

WITTWER, A. R., MÖLLER, S. V. (2000), Characteristics of the low speed wind tunnel of the UNNE, Journal of Wind Engineering \& Industrial Aerodynamics, 84, 307-320. 